\title{
Transport, Targeting and Applications of Functional Nanoparticles for Degradation of Chlorinated Organic Solvents
}

\author{
Gregory V. Lowry \\ Civil and Environmental Engineering \\ Carnegie Mellon University \\ 5000 Forbes Avenue \\ Pittsburgh, PA 15213
}

\section{Project Objectives}

This project addresses the need for methods to remove or degrade subsurface contaminants that are present as dense non-aqueous phase liquids (DNAPLs), and act as long-term sources of groundwater contamination. The goal is to build on a particle-based approach to subsurface contaminant remediation that is based partly on the recent success in using nanoparticle iron to degrade chlorinated compounds dissolved in groundwater, and knowledge of how colloids migrate in porous media. The objective is to engineer reactive nanoparticles that can decompose and potentially isolate DNAPL pollutants in the subsurface. Delivering reactive particles directly to the surface of the DNAPL will decompose the pollutant into benign materials, reduce the migration of pollutant during treatment, possibly lead to encapsulation of the DNAPL, and reduce the time needed to remove residual pollution by other means, such as natural attenuation.

The primary objectives of the project are to:

1. Design and synthesize reactive $\mathrm{Fe} 0$ and bimetallic $\mathrm{Fe} 0$ /catalyst particles that have surfaces modified with organic polymers such that the particles partition to/in DNAPLs.

2. Determine how variables such as size, composition, availability of water, and exposure to the transporting medium affect their ability to degrade TCE.

3. Investigate the transportability and water-DNAPL partitioning of particles in saturated porous media using micro-model and intermediate scale experiments in porous media. Determine how physical, chemical, and hydrologic regimes existing in the subsurface affect nanoparticle migration.

4. Test a method of targeted delivery of particles to DNAPLs particle suspensions in a benign organic medium with properties engineered to reflect those that govern DNAPL migration.

5. Develop a predictive numerical model for the transport and degradation of DNAPLs in bench-scale and meso-scale experiments.

\section{Research Progress and Implications}

This project was initiated September 15, 2002. Research has focused on objectives 1) designing and synthesizing inorganic-organic hybrid particles with the appropriate water solubility and TCE partitioning behavior, 2) determining the effects of particle composition and history on the ability to degrade TCE, and 5) developing the numerical models to predict transport and partitioning behavior of the nanoparticles. 1) Designing and synthesizing inorganic-organic hybrid particles with the appropriate water solubility and TCE partitioning behavior. The nanoparticles with hydrophobic-hydrophilic shell were synthesized by Atom Transfer Radical Polymerization (ATRP), which is well-known as a robust technique for control/living radical polymerization (refs 1-4). ATRP allows the synthesis of well defined materials with prescribed block length and narrow polydispersity. The hybrid nanoparticles consist of an inorganic core ( $\mathrm{SiO} 2$ or $\mathrm{Fe} 0$ ) surrounded by a hydrophobic inner shell and a hydrophilic outer shell. The role of the hydrophobic block is to protect the core against contact with water, while the hydrophilic part was designed 
to enable the transport of the particles through groundwater layers. Our studies showed that a ratio of $1 / 5$ to $1 / 10$ between hydrophobic and hydrophilic blocks is sufficient to assure protection to the inorganic core, and also to provide water solubility of the hybrid nanoparticles. Studies performed on unattached block copolymers showed that polystyrene (PSt) and poly(methyl methacrylate) (PMMA) are good candidates for the hydrophobic blocks. Sulfonated polystyrene (SPSt) was selected as the hydrophilic block due to its excellent water solubility. Other segments, such as methacrylate macromonomers with poly(ethylene oxide) side chains strongly adhered to silica due to the polyether blocks, obstructing the transport of the hybrid nanoparticles in the subsurface. We used inorganic-organic particles (silica core-organic (polymer) shell as model nanoparticles for transport studies. Ultimately, reactive polymer-modified Fe0 particles will be optimized and evaluated for transportability and reactivity. $\mathrm{SiO} 2$ particles are being used while synthesis methods for polymer-modified $\mathrm{Fe} 0$ nanoparticles are being developed and optimized. The synthesis of silica-supported initiators, starting from $20 \mathrm{~nm}$ silica dispersed in methyl ethyl ketone, was described elsewhere (ref 5). Using the ATRP initiators covalently attached to the silica surface, we formed polystyrene shell with $\sim 120 \mathrm{~nm}$ size via ATRP. The shell was further sulfonated using acetyl sulfate method (ref 6). The sulfonated nanoparticles $(\sim 90 \mathrm{~nm})$ were completely soluble in water. We synthesized other hybrid particles by polymerizing methyl methacrylate using silica initiators, and then by chain extension with styrene. Further, the silica-supported block copolymers underwent sulfonation, to allow the formation of water soluble nanoparticles even for block copolymers with higher molecular weights. Silicasupported nanoparticles with molecular weight of the PMMA segment $\mathrm{Mn}=2800 \mathrm{~g} / \mathrm{mol}$ and $\mathrm{Mn}=21,000$ $\mathrm{g} / \mathrm{mol}$ of PMMA-block-SPSt had larger hydrodynamic radii ranging from $~ 500 \mathrm{~nm}-1000 \mathrm{~nm}$ over the temperature range 25-50 C. Changes in hydrodynamic radius are likely due to polymer extension and/or particle aggregation. The nature of these changes is under investigation. Concerning the synthesis of Fe0 nanomaterials, we developed a technique for building hydrophobic-hydrophilic hybrids which consist of a short anchoring poly(methacrylic acid) block, a hydrophobic PMMA protective shell, and a hydrophilic SPSt outer block. The anchoring poly(methacrylic acid) block was obtained by hydrolysis of t-butyl groups from poly (t-butyl methacrylate), while the sulfonation reaction of polystyrene was performed following the same procedure as used for silica particles. The possibility of anchoring block copolymers on $\mathrm{Fe} 0$ nanoparticles by means of poly(methacrylic acid) (PMAA) units was confirmed from our experiments. Thus, when using triblock copolymers (PMAA)2300-(PMMA)2000-(SPSt)6300 we obtained water soluble $\mathrm{Fe} 0$ nanoparticles with a hydrodynamic radius of $\sim 700 \mathrm{~nm}$ for temperatures between $25-25 \mathrm{C}$. Changes in hydrodynamic radius are likely due to polymer extension and/or particle aggregation. The nature of these changes is under investigation. Work is in progress to fully characterize these particles, to optimize the synthesis technique, and to establish the optimum ratio between the anchoring part and hydrophobic and hydrophilic blocks. Implications. Appropriate hydrophobic and hydrophilic polymer blocks were identified and the first generation of inorganic-organic hybrid nanoparticles is now available for characterization and transport experiments. Characterization/testing of these particles will provide feedback to improve the next generation of particles. The ability to functionalize $\mathrm{SiO} 2$ and $\mathrm{Fe} 0$ particles has been demonstrated. Future work. The ratios of hydrophilic/hydrophobic blocks will be varied to better control the water solubility, transport, and particle protection provided by the hydrophobic block. The poly (methacrylic acid block size will be varied to optimize polymer attachment to iron particles. Methods to produce particles with Si-C polymer linkages are being developed. The Si-C bonds will be more stable that the Si-O-C bonds in the current polymer coated particles, improving the long term stability of these particles. A manuscript presenting the results from the development/characterization of functionalized $\mathrm{SiO} 2$ and $\mathrm{Fe} 0$ particles is in preparation. 2) Determining the effects of particle composition and history on the ability to degrade TCE : Effects of particle morphology and composition of $\mathrm{Fe} 0$-based nanoparticles on their reactivity with TCE Two types of iron nanoparticles (20-100 nm) were investigated, Reactive Nanoscale Iron Particles (RNIP, supplied by Toda American, Inc.) and Boron-doped Fe0 particles (Fe0/B) synthesized in our laboratory using the aqueous sodium borohydrate reduction method (ref 7). Both particle types are highly reactive and rapidly transform trichloroethylene (TCE) to nontoxic compounds. Fe0/B particles have a 30-fold higher reaction rate (15Lg-1d-1) than RNIP particles (0.5Lg-1d-1) under similar reaction conditions. The primary reaction products for Fe0/B particles are ethane (79\%), butane (12\%) and hexane (9\%), while for RNIP ethane (78\%) and ethene (22\%), at an initial TCE concentration of $4.4 \mathrm{mg} / \mathrm{L}$. At a high initial TCE concentrations $(170 \mathrm{mg} / \mathrm{L})$ acetylene becomes a significant reaction intermediate $(>50 \%)$ using RNIP particles. The product distribution using $\mathrm{Fe} 0 / \mathrm{B}$ particles did not change at higher initial TCE concentrations. These results suggest that different reaction mechanisms are occurring for each particle type. $\mathrm{Fe} 0 / \mathrm{B}$ particles are also more efficient (80\%) than RNIP particles (40\%) even though they use a less 
efficient electron transfer pathway. Efficiency is defined here as the fraction of electrons available from $\mathrm{Fe} 0$ oxidation that are used to reduce TCE. The Atomic Resolution Microscope (ARM) at the National Center for Electron Microscopy (Lawrence Berkeley National Lab) was used to examine the thickness and the composition of the surface layer formed on the particles before and after reactions. ARM clearly indicates a core/shell morphology. EDX indicates that Fe and $\mathrm{O}$ are the dominant chemical species present in the particles. $\mathrm{Na}$ is present in trace amounts (probably due to the $\mathrm{NaBH} 4$ and $\mathrm{NaOH}$ present during synthesis), but no other atoms atomic species are detected. Additionally, the ratio of Fe to O increases in the center of the particles relative to the outer shell of the particles so it is likely that the inner core consists of $\mathrm{Fe} 0$ and the outer shell some iron oxide phase. The exact chemical nature of these phases has not yet been determined. Reactivity of RNIP particles terminated with some of the core material intact, suggesting that the surface layer inhibits complete reaction of the particles. On the contrary, the core/shell appearance on the Fe0/B particles did not exist after reaction with TCE in water for 20 days. Based on the products distribution and the Fe0 consumed, both types of particles have a high selectivity ( 104) for TCE over H2O in a relatively high TCE concentration $(>140 \mathrm{mg} / \mathrm{L})$. Implications. Particles made form borohydride reduction of $\mathrm{Fe} 2+$ in water have several favorable properties relative to TCE degradation. Their ability to be stabilized in air and retain their activity makes handling and subsurface delivery easier. The efficiency of the $\mathrm{Fe} 0 / \mathrm{B}$ particles is substantially higher that that of particles provided by Toda America, however the cost to produce these particles is roughly 1 order of magnitude more ( $\$ 300 / \mathrm{kg}$ for Fe0/B vs. $\$ 15 / \mathrm{kg}$ for the Toda America particles.

\section{Future work}

Full characterization of each type of particle will be performed including EDX and EELS to obtain sufficient chemical information about the particles types. Particle properties will be correlated with their ability to dechlorinate TCE to optimize the properties of the iron nanoparticles. A manuscript presenting the factors affecting the efficiency and lifetime of various Fe0 particles is in preparation. 5) Developing the numerical models to predict transport and partitioning behavior of the nanoparticles. - The diffusion rates of nanoscale particles, particularly in inhomogeneous environments such as water/DNAPL interfaces, are sensitive to the microscopic particle structure. Their structure is challenging to fully characterize experimentally, so computer simulations on molecular length-scales (Brownian Dynamics (BD) simulations) are being conducted to describe the dynamics and structure of polymer-grafted metal nanoparticles. These simulations will complement experimental particle characterization and, ultimately, will provide parametric inputs for macroscale simulations of particle transport in porous media. BD simulations of homopolymers. The dynamics of homopolymers in solution were modeled using a beadspring model with a Pade approximation for the Inverse Langevin function. Simulation results for the structure and dynamics of polystyrene in a theta solvent during extensional flow with prior BD simulations (ref 8) yielded excellent agreement between the two calculations. BD simulations of nanoparticles. For our purposes, simulating the Brownian Dynamics of a solid nanoparticle in a solvent is somewhat more challenging than simply reproducing the known translational diffusivity of solid particles, since we aim to correctly describe the dynamics of polymers grafted to the particle. As a result, the description of the solid particle must reproduce the correct translational and rotational diffusivities. Furthermore, the rigid nature of the particle must be included in the simulation. These tasks were accomplished by performing BD simulations of a set of nodes distributed on the surface of the nanoparticle subject to distance constraints (ref 9). A Monte Carlo algorithm is used to define the positions of these nodes on the nanoparticles surface in a way that approximately maximizes the distances between nodes. This definition of the node position is a natural one for describing sites to which polymers can be grafted on the particle. An additional set of nodes not sitting on the true surface of the particle are also included in the description of the particle in order to give the composite set of nodes the same moment of inertia as the true rigid nanoparticle. With this multi-site description, BD simulations of the nanoparticle (with no grafted polymer present) exhibit the correct translational and rotational diffusivities for a solid particle in a homogeneous solvent. BD simulations of inorganic-organic hybrid nanoparticles. Bead-spring models of homopolymers grafted to solid nanoparticles have been converted to forms suitable for use in BD simulations of inorganic-organic hybrid nanoparticles, and preliminary simulations are being performed for solid nanoparticles with short homopolymer chains grafted to them. Implications. Measurements of the polymer structure and the translational diffusivity of the composite particle are providing information that can be closely related to the experimental data available from light scattering. Working in tandem with light scattering experiments, 
we expect to be able to develop our BD simulations to a level where they can provide quantitative information supporting choices of graft densities, polymer chain lengths, etc. within the overall context of this project. Future work. Hydrodynamic interactions will be included in the BD simulations so that we can compare model results with experiments using silica and iron particles with sulfonated polystyrene. The sulfonated polystyrene is charged and this can affect the dynamic light scattering results. Particle-silica and particle-organic interactions will then be incorporated into the model.

\section{References}

1. Wang, J. S.; Matyjaszewski, K. J. Am. Chem. Soc. 1995, 117, 5614.

2. Patten, T. E.; Xia, J.; Abernathy, T.; Matyjaszewski, K. Science 1996, 272, 866.

3. Matyjaszewski, K.; Wang, J. S., US 5,763,548, 1995.

4. Pyun, J.; Matyjaszewski, K. Chem. Mater. 2001, 13, 3436.

5. Pyun, J. Jia, S.; Kowalewski, T., Patterson, G. D.; Matyjaszewski, K. Macromolecules 2003, 36, 5094.

6. Y. Tran, P. Auroy, J. Am. Chem. Soc. 2001, 123, 3644.

7. Zhang, Wei-xian, J. Nanoparticle Research, 2003, 5(3-4), 323-332.

8. L. Li, R.G. Larson and T. Sridhar, J. Rheol., 44, p. 291 (2000).

9. J. Ryckaert, G. Ciccotti, and H. J. C. Berendsen, J. Computational Physics, 23, p. 327-341 (1977) 